\title{
PPARGC1A coding variation may initiate impaired NEFA clearance during glucose challenge
}

\author{
P. W. Franks • U. Ekelund $\cdot$ S. Brage $\cdot$ J. Luan • \\ A. J. Schafer $\cdot$ S. O'Rahilly • I. Barroso $\cdot$ N. J. Wareham
}

Received: 27 October 2006 / Accepted: 1 December 2006 / Published online: 10 January 2007

(C) Springer-Verlag 2007

\begin{abstract}
Aims/hypothesis The peroxisome proliferator-activated receptor gamma coactivator 1-alpha protein, encoded by the PPARGC1A gene, transcriptionally activates a complex pathway of lipid and glucose metabolism and is expressed primarily in tissues of high metabolic activity such as liver, heart and exercising oxidative skeletal muscle fibre. Ppargcla-null mice develop systemic dyslipidaemia and hepatic steatosis. In humans, NEFAs downregulate PPARGC1A expression in skeletal muscle. Furthermore, a
\end{abstract}

P. W. Franks $(\bowtie)$

Genetic Epidemiology and Clinical Research Group,

Department of Public Health and Clinical Medicine,

Section for Medicine, Umeå University Hospital,

Umeå, Sweden

e-mail: paul.franks@medicin.umu.se

P. W. Franks $\cdot$ U. Ekelund $\cdot$ S. Brage $\cdot$ J. Luan $\cdot$ N. J. Wareham MRC Epidemiology Unit,

Cambridge, UK

\section{A. J. Schafer}

Incyte,

Porter Drive,

Palo Alto, CA, USA

S. O'Rahilly

Departments of Clinical Biochemistry and Medicine,

Addenbrooke's Hospital,

Cambridge, UK

I. Barroso

Metabolic Disease Group, The Wellcome Trust Sanger Institute,

The Wellcome Trust Genome Campus,

Hinxton,

Cambridgeshire, UK common non-synonymous coding variant at PPARGC1A (Gly482Ser, rs8192678) is associated with decreased PPARGC1A mRNA levels and increased type 2 diabetes risk.

Materials and methods In a population-based sample of 691 healthy middle-aged Europids we assessed whether Gly482Ser is associated with levels of NEFA when fasting and in response to an oral glucose challenge. We also assessed the potential effect-modifying role of adipose tissue mass on these phenotypes.

Results After adjustment for age, sex, fat mass and fatfree mass, the Ser 482 allele associated with higher NEFA at $30 \mathrm{~min}$ and $2 \mathrm{~h}$ and with NEFA AUC (all values $p \leq 0.02$ ). Furthermore, suggestive evidence of interaction between fat mass and Gly482Ser was observed for fasting NEFA $(p=0.059)$. After stratification by level of obesity, genotype associations were observed in the obese for fasting NEFA $(p=0.028)$ and NEFA at $30 \min (p=0.013)$ and $2 \mathrm{~h}(p=0.002)$, and with NEFA AUC ( $p=0.005)$, but no significant associations were observed in lean individuals (all values $p>0.6$ ).

Conclusions/interpretation Our observations indicate that NEFA clearance is blunted following a glucose load in carriers of the PPARCG1A Ser482 allele. This association is augmented by obesity.

Keywords Body composition · Fatty acids · Genetics . Gly482Ser $\cdot$ PPARGC1A

\footnotetext{
Abbreviations

PPARGC1A peroxisome proliferator-activated receptor gamma coactivator 1-alpha
} 


\section{Introduction}

Peroxisome proliferator-activated receptor gamma coactivator 1-alpha (PPARGC1A) mRNA is expressed predominantly in tissues with high metabolic activity, the majority of which are rich in mitochondria. These include heart, exercising high-oxidative (type-I) skeletal muscle, brown fat, kidney, liver and brain, and other tissues such as white adipose [1].

In animals, Ppargcla silencing results in reduced oxidative capacity and metabolic derangements, which include hepatic steatosis, lower mitochondrial volume density in type I smooth muscle fibre, reduced mitochondrial respiration in muscle and liver, and growth retardation of heart and soleus muscle [2]. In the skeletal muscle of people with type 2 diabetes, the expression of PPARGC1Acoactivated genes is lower than in normal glucose-tolerant control subjects [3]. Furthermore, a common non-synonymous sequence variant at PPARGC1A (Gly482Ser) is associated with reduced PPARGC1A mRNA levels in skeletal muscle [4] and increased risk of type 2 diabetes [5]. Although animal data indicate a role for PPARGC1A in lipogenesis, only one small study has thus far reported on the association between Gly482Ser and NEFA levels [6], with two other studies reporting associations between Gly482Ser and cholesterol levels [7, 8].

The purpose of the present study was to assess the relationships between the common Gly482Ser non-synonymous variant at PPARGC1A with NEFA levels when fasted and following an oral glucose challenge in 691 healthy Europids. Furthermore, because the molecular effects of PPARGC1A may be downregulated by obesity [9] and the only published study of Gly482Ser and NEFA was conducted in obese individuals [6], we also assessed the effect-modifying role that adipose mass might have on the relationships between Gly482Ser and NEFA levels.

\section{Subjects and methods}

Selection of the participants and metabolic tests Volunteers were participants in the Medical Research Council Ely Study, a population-based cohort study in Ely, Cambridgeshire. The original sample without known type 2 diabetes was randomly recruited between 1990 and 1992 using a population-based sampling frame consisting of all 40- to 65-year-olds living in Cambridgeshire, UK. The data from this report emanate from individuals examined between 1994 and 1997 for whom all necessary clinical measurements and genotype data are available. All participants provided written informed consent. Ethical permission for the study was granted by the Cambridge Local Research Ethics Committee and the study was carried out in accordance with the principles of the Declaration of Helsinki as revised in 2000.

Volunteers attended the clinic at $0830 \mathrm{~h}$ having fasted since $2200 \mathrm{~h}$ the previous evening. Quantities of fat mass and fat-free mass were calculated using a standard impedance technique (Bodystat, Isle of Man, The British Isles) with participants dressed in lightweight clothing and without shoes. Following the collection of anthropometric data, a sample of fasting blood was taken and participants drank $75 \mathrm{~g}$ anhydrous glucose (BMS Laboratories, Beverley, Yorkshire, UK) dissolved in $250 \mathrm{ml}$ water over $2-$ 5 min. Further blood samples were taken at 30 and $120 \mathrm{~min}$. Plasma NEFA was determined enzymatically on the basis of the activity of acyl-CoA synthetase (Roche Molecular Biochemicals, Lewes, Sussex, UK). The resultant acyl-CoA is oxidised to yield hydrogen peroxide, which is measured colorimetrically. NEFA concentrations were measured in 0-, 30- and 120-min samples during the OGTT. NEFA AUC was calculated as a measure of the overall NEFA response during the OGTT and was defined as the area under the trapezoid described by the NEFA measurements at time 0,30 and $120 \mathrm{~min}$.
Table 1 Participant characteristics (means \pm SD) stratified by Gly482Ser genotype

Tests for difference by genotype were performed using linear regression (continuous traits) and Pearson's $\chi^{2}$ (categorical traits).

All analyses are unadjusted. Fasting insulin was $\log$ transformed prior to analysis

\begin{tabular}{|c|c|c|c|c|}
\hline & $\begin{array}{l}\text { Gly482Gly } \\
(n=268)\end{array}$ & $\begin{array}{l}\text { Gly482Ser } \\
(n=339)\end{array}$ & $\begin{array}{l}\text { Ser482Ser } \\
(n=84)\end{array}$ & $p$ value \\
\hline Age (years) & $55.3 \pm 10.5$ & $54.2 \pm 10.2$ & $55.4 \pm 10.5$ & 0.622 \\
\hline Sex ( $\%$ female $)$ & 62 & 57 & 61 & 0.418 \\
\hline Fat mass $(\mathrm{kg})$ & $24.4 \pm 8.3$ & $23.3 \pm 8.5$ & $23.4 \pm 7.5$ & 0.134 \\
\hline Fat-free mass $(\mathrm{kg})$ & $49.7 \pm 11.5$ & $51.0 \pm 11.1$ & $50.2 \pm 10.4$ & 0.367 \\
\hline Fasting NEFA (mmol/l) & $0.49 \pm 0.21$ & $0.49 \pm 0.22$ & $0.52 \pm 0.26$ & 0.275 \\
\hline 30-min NEFA (mmol/l) & $0.30 \pm 0.15$ & $0.31 \pm 0.16$ & $0.35 \pm 0.20$ & 0.066 \\
\hline 2-h NEFA (mmol/l) & $0.07 \pm 0.03$ & $0.07 \pm 0.05$ & $0.08 \pm 0.06$ & 0.075 \\
\hline NEFA AUC $\left(\mathrm{mmol} \mathrm{l}^{-1} \mathrm{~h}^{-1}\right)$ & $0.48 \pm 0.20$ & $0.48 \pm 0.22$ & $0.53 \pm 0.27$ & 0.047 \\
\hline Fasting insulin (pmol/l) & $49.7 \pm 37.4$ & $47.2 \pm 37.1$ & $43.5 \pm 28.0$ & 0.202 \\
\hline Fasting glucose $(\mathrm{mmol} / \mathrm{l})$ & $4.9 \pm 0.6$ & $4.9 \pm 0.6$ & $4.9 \pm 0.5$ & 0.308 \\
\hline 2-h glucose $(\mathrm{mmol} / \mathrm{l})$ & $5.7 \pm 1.8$ & $5.7 \pm 1.9$ & $5.7 \pm 1.8$ & 0.923 \\
\hline
\end{tabular}


Genetic analyses The methods for DNA extraction and genotyping of Gly482Ser in this cohort have been described in detail previously [10]. The genotype call rate was $>95 \%$.

Statistics All statistical analyses were conducted using SAS software V9.1 (SAS Institute, Carey, NC, USA). Generalised linear regression models were fitted to assess associations between genotypes and NEFA levels. Interaction analyses were undertaken by fitting a term for genotype $\times$ fat mass. Models were adjusted for age, sex, fat mass and fat-free mass. Central tendency and variance are reported as arithmetical means and SDs, respectively. A $p$ value $<0.05$ was considered statistically significant.

\section{Results}

Genotype frequencies for Gly482Ser were 39.2\% (Gly/Gly), 48.9\% (Gly/Ser), and 11.9\% (Ser/Ser). Genotype distributions conformed to Hardy-Weinberg predictions $(p>0.1)$.

Anthropometric, biochemical and body composition data are given in Table 1 stratified by genotype. With the exception of NEFAs, none of the comparisons between genotype groups approached statistical significance. The associations between Gly482Ser and insulin and glucose variables have been examined previously in these data [10].

After adjustment for age, sex, fat mass and fat-free mass, Gly482Ser was associated with NEFA at $30 \mathrm{~min}(p=0.02)$ and $2 \mathrm{~h}(p=0.01)$ and with NEFA AUC $(p=0.019)$. As shown in Fig. 1a, NEFA levels did not differ between genotypes when fasted but were higher in carriers of the minor Ser482 allele during the glucose challenge, and the allelic effects on NEFA were additive.

Linear regression models, fitted with an interaction term for Gly482Ser $\times$ fat mass and adjusted for age, sex, Gly482Ser, fat mass and fat-free mass, were used to test whether adiposity modifies the relationship between Gly482Ser and NEFA levels. In these models, we observed suggestive evidence for interaction on fasting NEFA $(p=0.059)$. We proceeded by stratifying the cohort below and above the sex-specific median for fat mass and re-tested within these groups for association between genotype and NEFA levels. The median age, fat mass $(\mathrm{kg})$, fat mass $(\%)$ and BMI were 56.5 years, $19.0 \mathrm{~kg}, 24.4 \%$ and $26.4 \mathrm{~kg} / \mathrm{m}^{2}$ for men, and 53.7 years, $24.9 \mathrm{~kg}, 37.6 \%$ and $25.4 \mathrm{~kg} / \mathrm{m}^{2}$ for women, respectively. As indicated in Fig. 1b, in lean individuals, NEFA levels are similar irrespectively of PPARCG1A genotype. However, in obese individuals (Fig. 1c), the association between genotype and NEFA levels is statistically significant at all time points, with the level of significance increasing with time during the OGTT.

\section{Discussion}

In the present study we report blunted NEFA clearance following glucose challenge in carriers of the minor PPARGC1A allele at Gly482Ser. This finding is consistent with previous studies suggesting that the Ser482 allele is associated with impairments in lipid oxidation [6] and that the carriers of this allele are more susceptible to type 2 diabetes than non-carriers [5]. We also observed interaction between genotypes and adipose mass on NEFA levels, where the associations between the Gly482Ser genotype

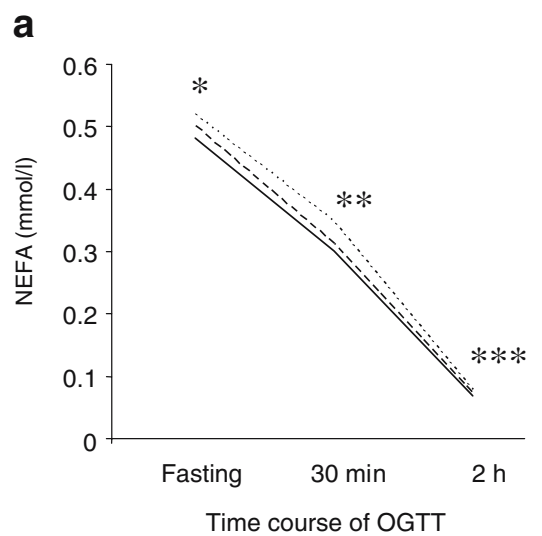

b

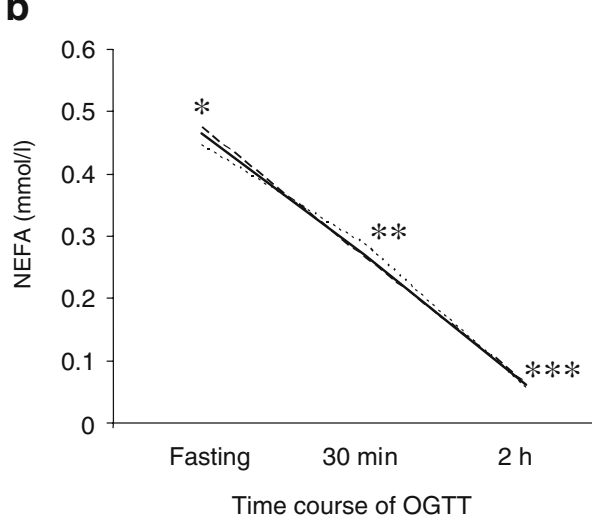

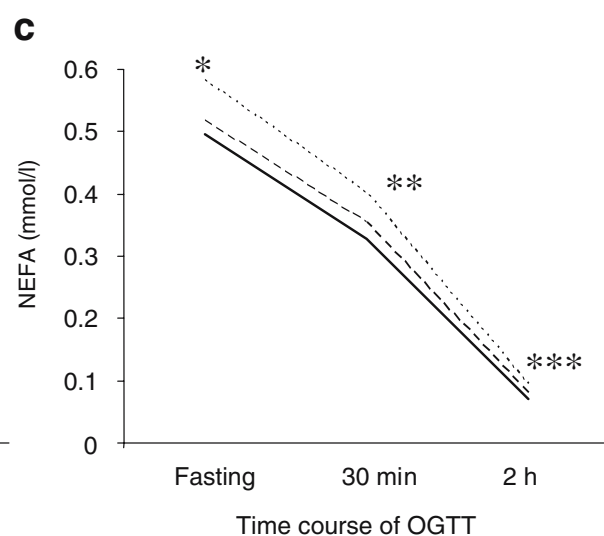

Fig. 1 NEFA levels during oral glucose challenge (75-g OGTT) stratified by Gly482Ser genotype in men and women: the Medical Research Council Ely Study ( $n=691)$. Solid lines, Gly482Gly; broken lines, Gly482Ser; dotted lines, Ser482Ser. a Data in the entire population; ${ }^{*} p=0.13,{ }^{*} p=0.02, * * * p=0.04$; NEFA AUC $p=0.019$. b Data stratified by lean $(n=351) ; * p=0.86 ; * * p=0.74 ; * * * p=0.64$; NEFA AUC $p=0.91$. c Data stratified by obese $(n=340) ;{ }^{*} p=0.28$;
$* * p=0.013 ; * * * p=0.002 . p$ values are from linear regression models, as reported in the Results, comparing NEFA levels for each genotype at different time points and are adjusted for age, sex, fat-free mass and fat mass. The median age, fat mass $(\mathrm{kg})$, fat mass (\%) and BMI were 51.5 years, $17.9 \mathrm{~kg}, 26.8 \%$ and $24.0 \mathrm{~kg} / \mathrm{m}^{2}$ for lean, and 58.6 years, $27.9 \mathrm{~kg}, 37.9 \%$ and $28.6 \mathrm{~kg} / \mathrm{m}^{2}$ for obese individuals, respectively 
and NEFA levels were consistently stronger in obese individuals than in their lean counterparts.

A number of studies have reported an association between the common Gly482Ser non-synonymous sequence variant at PPARGC1A and risk of type 2 diabetes. Using meta-analysis, we recently summarised the data from these studies and showed an $\sim 11 \%$ increased risk of diabetes per copy of the Ser482 allele [5]. In Pima Indians, Muller et al. [6] found no evidence for association between Gly482Ser and type 2 diabetes, but in a small sub-sample of obese non-diabetic Pimas $(n=139)$ observed lower fasting NEFA levels in carriers of the Ser482 allele compared with Gly482 allele homozygotes. Our findings, which contrast with these results, could be due to statistical fluctuation, meaning that either or both sets of results are false-positive, or that the Ser 482 allele is located within different ancestral haplotypes in Europids and Pima Indians. Alternatively, very pronounced and compensatory hyperinsulinaemia, which is a feature of the pre-diabetic state in Pimas and is less common in Europids [11], could perceivably explain this difference.

Ling et al. [4] studied the relationship between PPARGC1A mRNA levels and Gly482Ser genotype in non-diabetic Danish individuals, and reported lower PPARGC1A mRNA levels in older carriers of the Ser 482 allele compared with Gly482 homozygotes. However, Esterbauer et al. [8] found that the binding sites for known PPARGC1A transcription factors are not altered by this polymorphism, mitigating against a functional role for this variant. Although Gly482Ser may not causally relate to type 2 diabetes, this genotype has been associated with the disease in several studies [5].

In healthy individuals, NEFA levels decline postprandially. However, elevated NEFA when fasted and following food intake impedes glucose disposal via inhibition of insulin signalling, and promotes de novo hepatic lipid production, all of which play important roles in the development of type 2 diabetes. In white adipose tissue of obese individuals, PPARGC1A mRNA is expressed at substantially lower levels than in the tissue of lean individuals [9], suggesting that obesity may interfere with the activation of PPARGC1A in adipose. PPARGC1A downregulation in obesity may be related to elevated NEFA levels. Hoeks et al. [12] studied the effect of lipid infusion and consequent changes in plasma NEFA levels on PPARGC1A transcript levels in nine healthy males. In that study, the authors observed a reduction in PPARGC1A mRNA to $\sim 62 \%$ of basal levels, which they suggest was due to elevated plasma NEFA levels. Viewing these observations in the context of our own, it is plausible that PPARGC1A nucleotide variation increases the risk of type 2 diabetes via a vicious cycle involving NEFA metabolism and PPARGC1A mRNA downregulation (Fig. 2). This process would be consistent

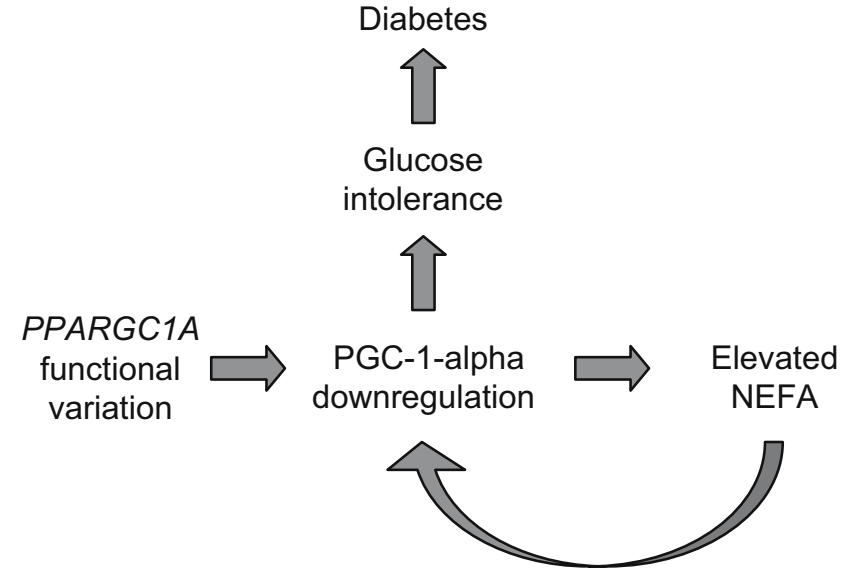

Fig. 2 Hypothetical model showing a vicious cycle that links the PPARGC1A gene with NEFA metabolism and impaired glucose homeostasis

with the development of chronic hyperglycaemia, which is often preceded by hyperlipidaemia, and may partially explain why obesity dramatically increases the risk of developing diabetes. Appropriately designed studies are required to test this hypothesis.

A key limitation of this study is that the sample size is relatively small. Thus, the level of statistical significance for our observations may be underestimated, and we cannot confidently conclude that our non-significant findings are truly negative. However, the test of interaction between fat mass and genotype provides reasonably strong evidence that the association between Gly482Ser genotype and NEFA levels is weaker in lean than in obese individuals.

In conclusion, the Gly482Ser variant at PPARGC1A may be associated with blunted NEFA clearance following an oral glucose challenge, associations that appear most evident in obese individuals. However, because our findings are novel and the associated level of statistical significance is modest, these findings require confirmation in other appropriately designed studies.

Acknowledgements We thank the volunteers who took part in this study. This study was funded by the UK Medical Research Council (N. J. Wareham). All genotyping was undertaken in collaboration with Incyte, Palo Alto, CA, USA. P. W. Franks was supported in part by grants from Novo Nordisk (370579201) and the Swedish Diabetes Association (DIA 2006-013).

Duality of interest No duality of interest is declared by any author.

\section{References}

1. Spiegelman BM, Puigserver P, Wu Z (2000) Regulation of adipogenesis and energy balance by PPARgamma and PGC-1. Int J Obes Relat Metab Disord 24(Suppl 4):S8-S10

2. Soyal S, Krempler F, Oberkofler H, Patsch W (2006) PGC1alpha: a potent transcriptional cofactor involved in the pathogenesis of type 2 diabetes. Diabetologia 49:1477-1488 
3. Mootha VK, Lindgren CM, Eriksson KF et al (2003) PGC1alpha-responsive genes involved in oxidative phosphorylation are coordinately downregulated in human diabetes. Nat Genet $34: 267-273$

4. Ling C, Poulsen P, Carlsson E et al (2004) Multiple environmental and genetic factors influence skeletal muscle PGC-1alpha and PGC-1beta gene expression in twins. J Clin Invest 114:1518-1526

5. Barroso I, Luan J, Sandhu M et al (2006) Meta-analysis of the Gly482Ser variant in PPARGC1A in type 2 diabetes and related phenotypes. Diabetologia 49:501-505

6. Muller YL, Bogardus C, Pedersen O, Baier L (2003) A Gly482Ser missense mutation in the peroxisome proliferator-activated receptor gamma coactivator-1 is associated with altered lipid oxidation and early insulin secretion in Pima Indians. Diabetes 52:895-898

7. Ek J, Andersen G, Urhammer SA et al (2001) Mutation analysis of peroxisome proliferator-activated receptor-gamma coactivator-1 (PGC-1) and relationships of identified amino acid polymorphisms to type II diabetes mellitus. Diabetologia 44:2220-2226
8. Esterbauer H, Oberkofler H, Linnemayr V et al (2002) Peroxisome proliferator-activated receptor-gamma coactivator-1 gene locus: associations with obesity indices in middle-aged women. Diabetes 51:1281-1286

9. Semple RK, Crowley VC, Sewter CP et al (2004) Expression of the thermogenic nuclear hormone receptor coactivator PGC1 alpha is reduced in the adipose tissue of morbidly obese subjects. Int J Obes Relat Metab Disord 28:176-179

10. Barroso I, Luan J, Middelberg RP et al (2003) Candidate gene association study in type 2 diabetes indicates a role for genes involved in beta-cell function as well as insulin action. PLoS Biol 1:E20

11. Lillioja S, Nyomba BL, Saad MF et al (1991) Exaggerated early insulin release and insulin resistance in a diabetes-prone population: a metabolic comparison of Pima Indians and Caucasians. J Clin Endocrinol Metab 73:866-876

12. Hoeks J, Hesselink MK, Russell AP et al (2006) Peroxisome proliferator-activated receptor-gamma coactivator-1 and insulin resistance: acute effect of fatty acids. Diabetologia 49:2419-2426 